\title{
Effet de la vasopressine et de l'indométhacine sur la diurèse provoquée chez Meriones crassus gravide et non gravide
}

\author{
K Baddouri, A Quyou \\ Université Mohammed $V$, faculté des sciences, laboratoire de physiologie animale \\ unité d'endocrinologie comparée, BP 1014 Rabat, Maroc
}

(Reçu le 2 avril 1990; accepté le 7 février 1991)

\begin{abstract}
Résumé - La cinétique de la diurèse provoquée a été étudiée chez une espèce de rongeur désertique Meriones crassus et au cours de la gestation. En réponse à une hydratation, les Meriones crassus gravides excrètent l'eau en excès plus rapidement que les femelles non gravides. L'installation de la diurèse est plus précoce chez les femelles gravides traduisant une inactivation plus rapide de la vasopressine. L'interaction probable de la vasopressine et des prostaglandines et son effet sur l'excrétion rénale pendant la gestation ont été montrés au cours d'un traitement à l'arginine-vasopressine et après inhibition de l'hormone endogène et des prostaglandines. Les résultats montrent une diminution de la réponse du rein à la vasopressine endogène durant la gestation chez Meriones crassus dont l'origine pourrait être l'augmentation du taux des prostaglandines dans le rein.
\end{abstract}

rongeur désertique / vasopressine / prostaglandine / diurèse / gestation

Summary - Effect of vasopressin and indomethacin on provoked diuresis in pregnant and non-pregnant Meriones crassus. The dynamics of induced diuresis was studied in Meriones crassus, a desert rodent species, during estrus and pregnancy. In response to hydration, pregnant $\mathrm{Me}$ riones crassus excrete excess water more rapidly than non pregnant females. Diuresis appears earlier in pregnant females indicating a more rapid inactivation of vasopressin. The possible interaction between vasopressin and prostaglandins, and its effect on renal excretion during pregnancy, was studied during arginine-vasopressin treatment and after inhibition of endogenous AVP and prostaglandins. Results show a decrease in renal response to endogenous vasopressin during pregnancy in Meriones crassus, which may be due to the increase in renal prostaglandin levels. 


\section{INTRODUCTION}

L'hormone antidiurétique joue un rôle fondamental dans le maintien de l'équilibre hydrique des mammifères. Cependant, chez Meriones crassus, un rongeur désertique qui ne consomme pas d'eau de boisson, le métabolisme hydrique est profondément modifié au cours de la gestation: on observe une augmentation du volume extracellulaire sans changement de la concentration d'arginine vasopressine (AVP) circulante mais avec une baisse de la pression osmotique plasmatique (Baddouri et Quyou, 1991). Ce phénomène est-il dû à une chute des seuils osmotiques de sensibilité à l'AVP ou bien à une augmentation de la résistance rénale à l'AVP sous l'effet des prostaglandines E (Roman et Lechene, 1982) ? Pour éclaircir ce point, ce travail rapporte les résultats de l'excrétion rénale chez des Meriones gravides et non gravides traitées par de l'AVP synthétique (pitressine), après inhibition de l'hormone endogène par surcharge hydrique, suivie on non d'un inhibiteur de synthèse des prostaglandines (indométhacine).

\section{MATÉRIEL ET MÉTHODES}

\section{Animaux et conditions expérimentales}

Nous avons utilisé des Meriones crassus adultes, petits rongeurs sahariens de la famille des gerbillidae. Ils recevaient des graines d'orge et de tournesol avec, comme seul apport d'eau, $5 \mathrm{~g}$ de salade par animal et par $24 \mathrm{~h}$.

Le cycle cstral dure en moyenne $3 \mathrm{j}$ avec un œstrus de $18 \mathrm{~h}$. La gestation dure $22 \mathrm{j}$ et le nombre de portées dépasse rarement 4 avec une prolificité moyenne de 3 petits par portée.

L'expérimentation a porté sur 5 femelles témoins (en œstrus) et 5 femelles en dernière se- maine de gestation $\left(19^{\mathrm{e}} \mathrm{j}\right)$ de poids moyens respectifs de $81 \pm 5$ et $93 \pm 2 \mathrm{~g}$.

\section{Hydratation des animaux}

Les animaux ont été préalablement adaptés pendant $3-4 \mathrm{j}$ à recevoir de l'eau par tubage gastrique (2-3\% du poids corporel). Avant l'expérimentation, les animaux étaient maintenus à jeûn pendant au moins $12 \mathrm{~h}$ et une légère compression de la vessie permettait d'éliminer l'urine préexistante juste avant la surcharge hydrique. Pour l'expérimentation ils recevaient en moins d'une min une quantité d'eau équivalente à $5 \%$ du poids du corps au moyen d'une pompe à perfusion $\left(5 \mathrm{ml} \cdot \mathrm{min}^{-1}\right)$. Chaque animal était ensuite placé dans une cage métabolique; les urines étaient récoltées dans des tubes tarés avec changement de tube et enregistrement du temps à chaque miction. L'expérience s'est poursuivie jusqu'à l'élimination totale de la surcharge hydrique. Les volumes d'urine ont été déterminés par pesée et la pression osmotique a été mesurée par cryométrie dans chaque échantillon urinaire. Les résultats sont exprimés par le temps de latence (délai entre le gavage et le début de la diurèse), le pouvoir d'excrétion (temps pour excréter $50 \%$ de la surcharge), le débit urinaire (en \% de la surcharge/min) et la pression osmotique au maximum de diurèse.

\section{Traitement à la vasopressine}

La même expérience a été répétée $24 \mathrm{~h}$ plus tard mais cette fois, immédiatement à la fin du gavage hydrique, les animaux ont reçu une injection intrapéritonéale de vasopressine (AVP) (pitressine Sigma, $1 \mathrm{mU} / 100 \mathrm{~g}$ de poids corporel). Les animaux témoins ont reçu une injection de solvant sans AVP.

\section{Inhibition des prostaglandines}

Le même type d'expérience a été réalisé avec l'injection intrapéritonéale complémentaire d'indométhacine $(25 \mathrm{mg} / \mathrm{kg}$ ) aussitôt après celle d'AVP. (Des essais préliminaires avaient montré 
un effet significatif de cette dose d'inhibiteur de synthèse des prostaglandines).

\section{Analyse statistique des résultats}

Les résultats sont exprimés en moyenne \pm ESM (erreur standard à la moyenne). La validité statistique des différences entre les moyennes de 2 séries expérimentales est évaluée d'après le test «t» de Fisher-Student. La différence entre les moyennes est considérée comme statistiquement significative au seuil de $5 \%(P<0,05)$.

\section{RÉSULTATS}

\section{Exploration rénale chez Meriones crassus gravides et non gravides}

Les paramètres urinaires montrent que chez les animaux non gravides le temps

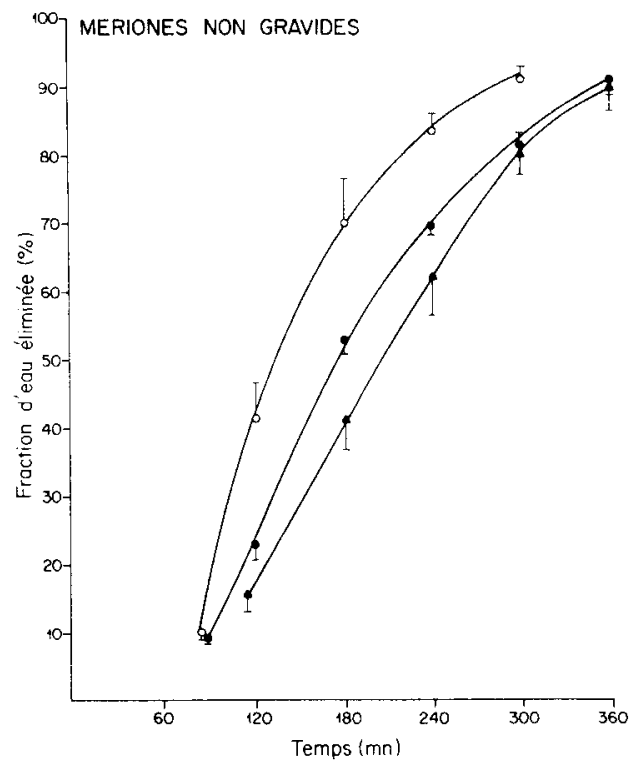

de latence est double de celui des animaux gravides (tableau I et fig 1). De plus dans les conditions de non gravidité, $M e-$ riones crassus excrète la totalité de la surcharge de l'eau dans un délai de $5 \mathrm{~h}$ environ (fig 1) et le débit urinaire mesuré pendant la phase de diurèse maximale (lorsque les urines ont l'osmolalité la plus faible, tableau II) n'atteint pas $1 \% / \mathrm{min}$ $(0,87 \pm 0,10 \% / \mathrm{min}$, tableau II). De ce fait, le pouvoir d'excrétion de ces animaux est plus faible que celui des femelles gravides $(136 \pm 22$ contre $88 \pm 5$; tableau I).

\section{Effet du traitement à l'AVP}

L'injection de l'AVP entraîne un retard de la réponse diurétique et une diminution du pouvoir d'excrétion chez les 2 groupes d'animaux. L'intensité de la réponse au traitement à l'AVP est équivalente chez les

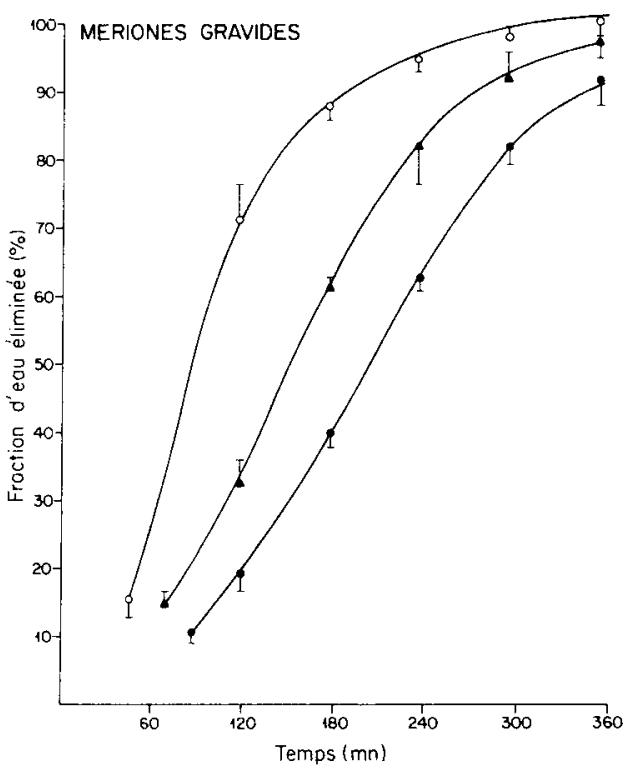

Fig 1. Cinétique d'élimination d'une surcharge hydrique ( $5 \%$ du poids corporel) après traitements pharmacologiques chez Meriones crassus gravides et non gravides. ( $m \pm$ esm, 5 animaux par lot); $O$ animaux témoins; $\wedge$ animaux traités à la vasopressine; $\bullet$ animaux traités à la vasopressine et à l'indométhacine. 
Tableau I. Caractéristiques de la diurèse induite par une surcharge hydrique (5\% du poids corporel) chez Meriones crassus gravide et non gravide. Moyennes \pm esm. Pour chaque condition expérimentale, les résultats sont significativement différents pour chacun des paramètres de l'excrétion urinaire entre animaux non gravides et gravides; $n=$ nombre d'animaux.

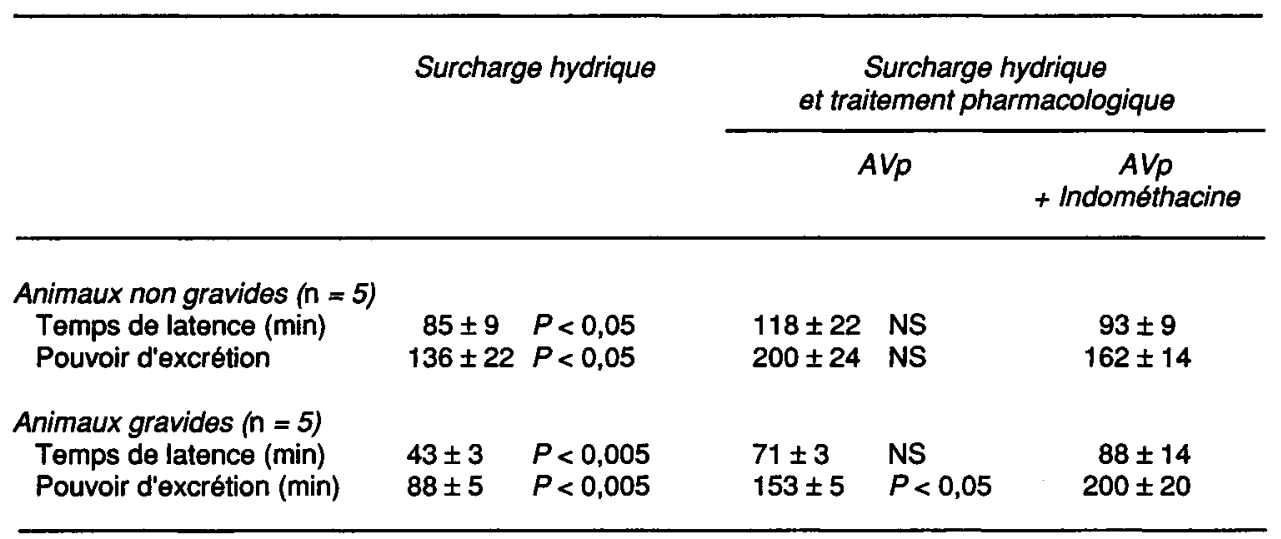

animaux gravides et non gravides (tableau I).

\section{Excrétion urinaire après inhibition des prostaglandines}

L'indométhacine ne modifie pas significativement la réponse à l'AVP des animaux non gravides (tableaux I et II). Elle ne retarde pas non plus la diurèse après traitement à l'AVP des Meriones gravides. Par contre, chez ces dernières le pouvoir d'excrétion et le débit urinaire sont significativement réduits par l'indométhacine (tableaux I et II).

Chez les 2 groupes d'animaux les osmolalités urinaires correspondant à la phase maximale de la diurèse sont significativement augmentées par le traitement à l'AVP et l'indométhacine ne modifie pas cette réponse (tableau II).

\section{DISCUSSION}

Les résultats montrent clairement une réponse diurétique plus rapide et plus importante à la suite d'une surcharge hydrique ( $5 \%$ du poids corporel) chez les Meriones gravides que chez les femelles non gravides. La légère différence du volume d'eau reçu par les femelles gravides $(4,5$ $\mathrm{ml}$ contre $4 \mathrm{ml}$ chez les non gravides) ne peut expliquer à elle seule les résultats.

Au cours de la réponse diurétique (fig 1) chez les 2 groupes de Meriones l'excrétion de la moitié de la surcharge d'eau correspond à la période de diurèse maximale, c'est à dire au moment où les urines ont l'osmolalité la plus basse : chez les $\mathrm{Me}$ riones gravides, non seulement le temps de latence de l'excrétion urinaire, mais aussi le délai nécessaire à l'élimination de $50 \%$ de la surcharge d'eau sont significativement plus courts. Ces résultats ne peu- 
Tableau II. Pression osmotique et débit urinaire au cours d'une surcharge hydrique et après injection de vasopressine et d'inhibiteur des prostaglandines chez Meriones crassus gravide et non gravide. Moyennes \pm esm. Les débits urinaires mesurés chez les animaux gravides sont significativement plus élevés $(P<0,05)$ que ceux mesurés chez les animaux non gravides. Par contre, au cours du traitement pharmacologique, les valeurs sont identiques chez les 2 lots d'animaux. L'osmolalité urinaire correspond à la valeur mesurée pendant la période de dilution maximale de l'urine; $n=$ nombre d'animaux.

$\begin{array}{cc}\text { AVp } & \text { AVp } \\ + & \text { indométhacine }\end{array}$

\begin{tabular}{|c|c|c|c|c|c|}
\hline \multicolumn{6}{|c|}{ Animaux non gravides $(\mathrm{n}=5)$} \\
\hline $\begin{array}{l}\text { Débit urinaire (\%.min-1) } \\
\text { Pression osmotique }\end{array}$ & $0,87 \pm 0,10$ & $P<0,005$ & $0,54 \pm 0,07$ & NS & $0,60 \pm 0,07$ \\
\hline max diurèse (mosm. $\left.\mathrm{kg}^{-1}\right)$ & $131 \pm 11$ & $P<0,05$ & $167 \pm 15$ & NS & $188 \pm 20$ \\
\hline \multicolumn{6}{|l|}{ Animaux gravides $(n=5)$} \\
\hline $\begin{array}{l}\text { Débit urinaire (\%.min-1) } \\
\text { Pression osmotique } \\
\text { (mosm. } \text { kg }^{-1} \text { ) }\end{array}$ & $\begin{array}{l}1,04 \pm 0,13 \\
120 \pm 7\end{array}$ & $\begin{array}{l}P<0,05 \\
P<0,005\end{array}$ & $\begin{array}{l}0,55 \pm 0,03 \\
210 \pm 12\end{array}$ & $\begin{array}{l}P<0,05 \\
\text { NS }\end{array}$ & $\begin{array}{l}0,44 \pm 0,075 \\
223 \pm 15\end{array}$ \\
\hline
\end{tabular}

vent être expliqués par une clairance métabolique augmentée durant la gestation notamment par les vasopressinases d'origine placentaire, car, d'une part il a été rapporté que les rongeurs ne produisent pas cette enzyme (Rosembloom et al, 1975) et d'autre part la concentration plasmatique de cette hormone ne varie pas au cours de la gestation (Baddouri et Quyou, 1991). Dans le rein, les prostaglandines de type $E$ inhibent l'action de l'AVP (Dunn et al, 1982; Roman et Lechene, 1982) et leurs synthèses augmentent pendant la gestation chez certaines espèces (Gant et al, 1980). Ces hormones augmentent le débit urinaire par un effet hémodynamique (Andersson et al, 1976) et/ou en inhibant la production de l'AMPc - AVP dépendante dans le canal collecteur du rein (Torikai et Kurokawa, 1983). L'inhibition de la synthèse des prostaglandines par l'indométhacine ne modifie pas la réponse du rein à I'AVP chez les Meriones non gravides, par contre chez les femelles gravides le temps nécessaire à l'excrétion de $50 \%$ de la surcharge, avec une dilution maximale des urines, augmente et devient similaire à celui des animaux non gravides traités à l'AVP seule (200 min). La différence de réponse entre ces 2 groupes d'animaux correspond au temps nécessaire à la dégradation de l'AVP endogène chez les femelles gravides traitées à l'indométhacine puisque durant la diurèse maximale, les osmolalités urinaires sont identiques chez les femelles gravides traitées à l'AVP et chez celles qui ont reçu de l'AVP et l'inhibiteur des prostaglandines.

La stimulation de la diurèse pendant la gestation a été décrite chez d'autres espèces de Mammifères (Olsson et al, 1982) chez lesquelles une polydipsie a été observée. En effet, l'augmentation de la synthèse des prostaglandines au niveau du système nerveux central stimule la soif (Barron et al, 1984). Il faut donc admettre chez Meriones crassus, espèce qui ne consomme pas d'eau de boisson, l'exis- 
tence possible d'une adaptation de la fonction rénale qui permettrait de limiter les pertes en eau comme chez d'autres espèces de rongeurs désertiques (Baddouri et al, 1987), particulièrement pendant la gestation. Il est probable que la concentration des prostaglandines rénales est relativement faible chez cette espèce. Dans ces conditions, seule une fraction de l'AVP endogène serait inhibée durant la gestation comme le suggère le traitement à la vasopressine qui induit une réponse équivalente chez les Meriones gravides et non gravides où l'excrétion de la moitié de la surcharge est retardée d' $1 \mathrm{~h}$ environ chez les 2 groupes d'animaux. Cependant l'indométhacine possède des actions indépendantes des prostaglandines, telle l'inhibition compétitive de l'aldostérone pour les récepteurs minéralocorticoïdes et la diminution de l'effet biologique de l'angiotensine II liée aux cellules surrénales (Dunn et Zambraski, 1980). II n'est donc pas exclu que l'indométhacine puisse agir par un effet natriurétique et donc diurétique. L'utilisation d'autres inhibiteurs des prostaglandines qui dissocieraient les actions non spécifiques et non prostaglandiniques de l'indométhacine permettrait de préciser les interprétations.

\section{RÉFÉRENCES}

Andersson RJ, Berl T, Mc Donald KM, Schrier RW (1976) Prostaglandins: effect on blood pressure, renal blood flow, sodium and water excretion. Kidney Int 10, 205-215
Baddouri KH, Quyou A (1991) Osmolalité et sécrétion de vasopressine au cours de la gestation chez Meriones crassus. Reprod Nutr Dev. Soumis à publication

Baddouri KH, El Hilali M, Marchetti J, Menard J (1987) Renal excretion capacity in hydrated desert rodents (Jaculus orientalis and Jaculus deserti) J Comp Physiol B 157, 237-240

Barron MW, Stamoutros $A B$, Lindheimer DM (1984) Rôle of volume in the regulation of vasopressin secretion during pregnancy in the rat. J Clin Invest 73, 923-932

Dunn M, Zambraski EJ (1980) Renal effects of drugs that inhibit prostaglandin synthesis. Kidney Int 18, 609-622

Dunn M, Pugliese F, Aikawa M, Williams S, Hassid A (1982) Rabbit and renal papillary collecting tubule (RPCT) cells in culture: the interactions of arginine vasopressin (AVP), prostaglandins (PGs), and cyclic AMP (CAMP). Kidney Int 21, 259 (1 page)

Gant FN, Worley JR, Everett BR, Mc Donald PC (1980) Control of vascular responsiveness during human pregnancy. Kidney Int 18, 253258

Olsson K, Benlamlih S, Dahlborn K, Fyhrquist F (1982) Effects of water deprivation and hyperhydration in pregnant and goats. Acta Physiol Scand 115, 316-367

Roman RJ, Lechene C (1982) Renal prostaglandins inhibit tubular reabsorption of $\mathrm{NaCl}$, and $\mathrm{Mg}$ in antidiurectic and water diuretic rats. Kidney Int 21, 264 (1 page)

Rosembloom AA, Sack J, Fisher DA (1975) The circulating vasopressinase of pregnancy: species comparaison with radio-immunoassay. Am J Obstet Gynecol 121, 316-320

Torikai S, Kurokawa K (1983) Effect of $\mathrm{PGE}_{2}$ on vasopressin-dependent cell CAMP in isolated single nephron segments. Am J Physiol 245 (Renal Fluid Electrolyte Physiol 14): F 58F66 